\title{
Hypercompact H II Regions: Resolved Images of G34.26+0.15 A and B
}

\section{Citation}

Avalos, Martín, Susana Lizano, Ramiro Franco-Hernández, Luis F. Rodríguez, and James M. Moran. 2008. "Hypercompact H II Regions: Resolved Images of G34.26+0.15 A and B" The Astrophysical Journal 690 (2) (December 2): 1084-1088. doi:10.1088/0004-637x/690/2/1084.

\section{Published Version}

doi:10.1088/0004-637X/690/2/1084

\section{Permanent link}

http://nrs.harvard.edu/urn-3:HUL.InstRepos:26519151

\section{Terms of Use}

This article was downloaded from Harvard University's DASH repository, and is made available under the terms and conditions applicable to Other Posted Material, as set forth at http:// nrs.harvard.edu/urn-3:HUL.InstRepos:dash.current.terms-of-use\#LAA

\section{Share Your Story}

The Harvard community has made this article openly available.

Please share how this access benefits you. Submit a story.

Accessibility 


\title{
HYPERCOMPACT H II REGIONS: RESOLVED IMAGES OF G34.26+0.15 A AND B
}

\author{
Martín Avalos $^{1}$, Susana Lizano ${ }^{1}$, Ramiro Franco-Hernández ${ }^{1,2}$, Luis F. Rodríguez ${ }^{1}$, And James M. Moran ${ }^{2}$ \\ ${ }^{1}$ Centro de Radioastronomía y Astrofísica, UNAM, Apdo. Postal 72-3 (Xangari), 58089 Morelia, Michoacán, Mexico; m.avalos@astrosmo.unam.mx. \\ ${ }^{2}$ Harvard Smithsonian Center for Astrophysics, 60 Garden Street, Cambridge, MA 02138, USA \\ Received 2008 April 29; accepted 2008 August 27; published 2008 December 2
}

\begin{abstract}
We report high angular resolution observations at $\lambda=7 \mathrm{~mm}$ of the hypercompact $(\mathrm{HC}) \mathrm{H}$ II regions $\mathrm{G} 34.26+0.15$ $\mathrm{A}$ and $\mathrm{B}$. The images and the intensity profiles obtained give possible evidence of limb brightening, which may indicate the existence of inner "holes" in the ionized gas. These "holes" were previously inferred from a study of the spectra and angular sizes at different frequencies from lower angular resolution observations. Using a spherical ionized model bounded by an inner and an outer radii with an electron density gradient $n_{e} \propto r^{-\alpha}$, we can reproduce the $7 \mathrm{~mm}$ intensity profile, spectral energy distribution, and observed angular sizes of both sources. These models indicate inner radii $R_{1} \sim 400 \mathrm{AU}$, outer radii $R_{2} \sim 1000 \mathrm{AU}$, and shallow density gradients with slopes $\alpha \sim 0.3-1.0$. These profiles are similar in physical size and shape to those found recently for the $\mathrm{HC} \mathrm{HII}$ regions G24.78+0.08 A1 and G28.20-0.04N. Therefore, at least four HC Hil regions seem to have thick shells of ionized gas with fractional widths, $\left(R_{2}-R_{1}\right) / R_{2} \sim 0.1-0.6$, in contrast with the thin ionized shells expected in the case of shells swept up by stellar winds. More observations are needed to determine the origin of these thick ionized shells and their frequency of occurrence in the HC HII region stage.
\end{abstract}

Key words: H II regions - radiative transfer - radio continuum: ISM - stars: formation

\section{INTRODUCTION}

In recent years, observational and theoretical effort has been devoted to understanding the process of massive star formation. Hypercompact (HC) H II regions seem to correspond to the earliest stage of this process. HC $\mathrm{H}$ II regions are very small ionized regions around massive stars, with sizes of $L \lesssim 0.01 \mathrm{pc}$ and emission measures of $\mathrm{EM} \gtrsim 10^{8} \mathrm{~cm}^{-6} \mathrm{pc}$, implying turnover frequencies greater than or equal to $5 \mathrm{GHz}$. They have rising continuum spectra $S_{v} \propto v^{\alpha}$, with $\alpha \sim 1$, and many of them have broad radio recombination lines with $\Delta v>40 \mathrm{~km} \mathrm{~s}^{-1}$ (e.g., Sewiło et al. 2004). It is important to fully determine their physical properties, such as density, size, morphology, and kinematics, in order to understand this stage in the evolution of H II regions (e.g., Hoare et al. 2007). The physical properties of these regions may have useful information about the conditions in which massive stars are formed and the processes that occur during their very early phases.

G34.26+0.15 is a star formation complex at a distance of $3.3 \mathrm{kpc}$. It is composed of four $\mathrm{H}$ II regions at different evolutionary stages (sources A, B, C, and D) and two infrared sources (sources E and F) reported by Campbell et al. (2000). G34.26+0.15 C is a cometary ultracompact $\mathrm{H}$ II region, responsible for most of the emissions at radio wavelengths. G34.26+0.15 $\mathrm{A}$ and $\mathrm{B}$ are the two $\mathrm{HC} \mathrm{HII}$ regions located to the east of source $\mathrm{C}$.

Avalos et al. (2006, hereafter A2006) studied the spectra and angular sizes at different frequencies of sources A and B. These sources are on the lower-density end of $\mathrm{HC} \mathrm{H}$ II regions and have a turnover frequency of $v \sim 10 \mathrm{GHz}$. From the optically thin radio emission of both sources A2006 obtained an emission measure EM $\sim 1-2 \times 10^{8} \mathrm{~cm}^{-6} \mathrm{pc}$. Also, the rate of ionizing photons for both sources is $\dot{N} \sim 2 \times 10^{47} \mathrm{~s}^{-1}$ which corresponds to exciting stars with spectral types earlier than the B1 type (according to Vacca et al. 1996) or the B0 type (according to Panagia 1973). A2006 compared the radio continuum spectra and angular sizes of sources A and B with a simple model of spherical regions bounded by an inner and an outer radii ( $R_{1}$ and $R_{2}$, respectively), with a power-law electron density profile, $n_{e}(r) \propto r^{-\alpha}$. They found that the spectra and sizes could be reasonably reproduced by either uniform sphere models, or by shell models with large inner radii, of the order of $500 \mathrm{AU}$. For models with a power-law electron density an inner hole is necessary, otherwise, the gas would remain optically thick at high frequencies, due to the divergence of the density at the origin.

Unfortunately, from the observed angular sizes it was not possible to distinguish between these models. High-resolution observations at $v>15 \mathrm{GHz}$ were proposed in order to distinguish between them. If the source could be resolved, then the presence of inner "holes" would show up as a "limb brightening." Also, intensity profiles would provide a strong constraint on models and allow discrimination among different density profiles.

In this work we present high-resolution $7 \mathrm{~mm}$ observations of G34.26+0.15 A and B. Resolved intensity profiles of both sources give evidence in favor of a limb brightening that indicates the presence of inner "holes." This paper is organized as follows. In Section 2, we discuss the observations. In Section 3, we present the bounded ionized power-law models that fit the $7 \mathrm{~mm}$ intensity profiles, and the spectra and sizes of both sources. In Section 4 we discuss our results. Finally, in Section 5 we give our conclusions.

\section{OBSERVATIONS}

The $7 \mathrm{~mm}$ observations were made in the A configuration of the Very Large Array (VLA) of the $\mathrm{NRAO}^{3}$, on 2006 April 2. The central frequency observed was $43.34 \mathrm{GHz}$ and we integrated on-source for a total of $2.2 \mathrm{~h}$. The absolute amplitude calibrator was $0137+331$ (with an adopted flux density of $0.53 \mathrm{Jy}$ ) and the

\footnotetext{
3 The National Radio Astronomy Observatory is operated by Associated Universities Inc. under cooperative agreement with the National Science Foundation.
} 


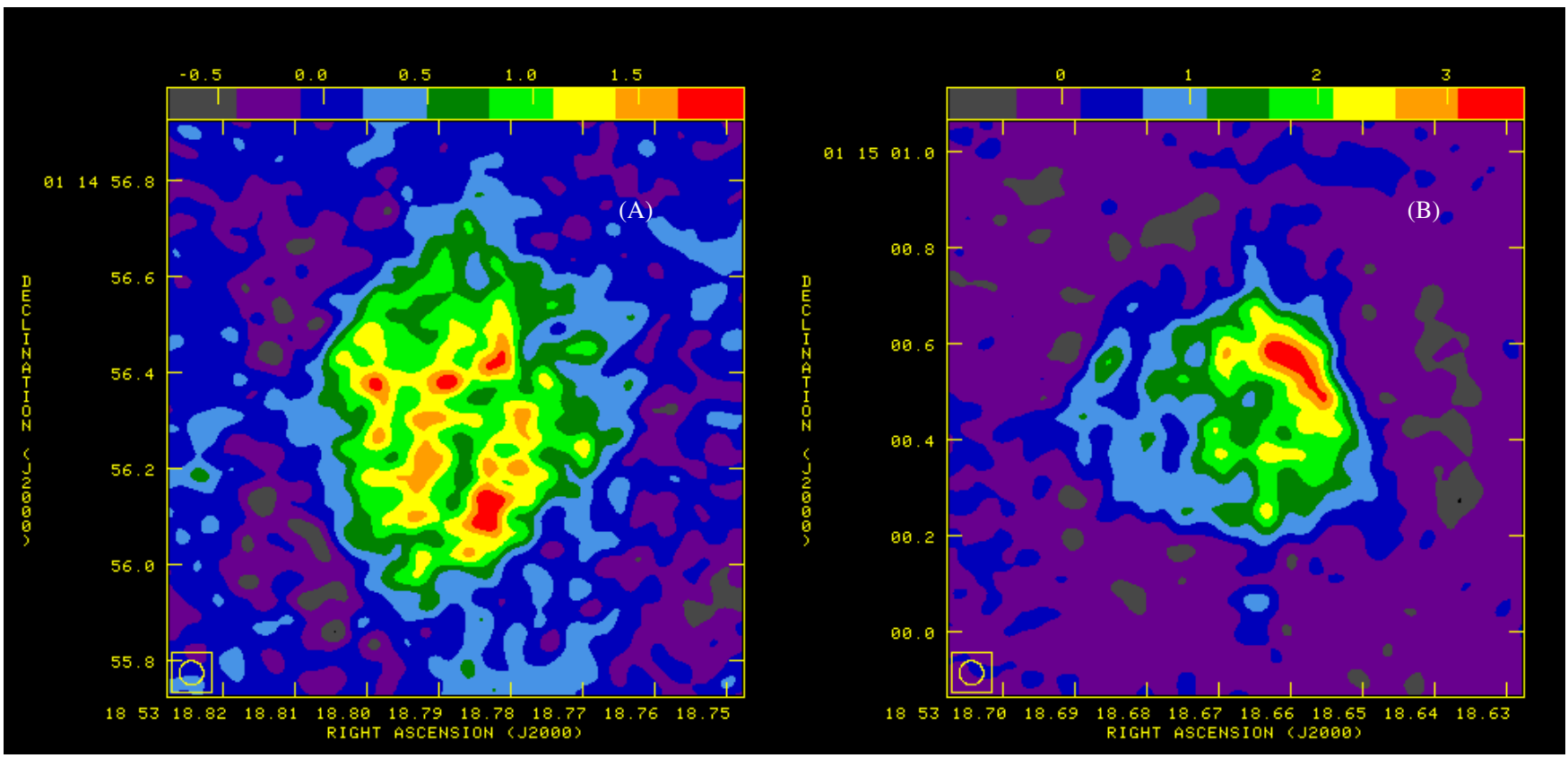

Figure 1. Color images of G34.26+0.15 A (left) and B (right) made using the VLA $7 \mathrm{~mm}$ observations. The color bar on top gives the flux density per beam in units of $\mathrm{mJy}_{\text {beam }}{ }^{-1}$. The half-power contour of the beam is shown in the bottom-left corner.

phase calibrator was $1851+005$ (with a bootstrapped flux density of $0.94 \pm 0.02 \mathrm{Jy})$. The amplitude calibrator was self-calibrated in amplitude and phase using a model provided by NRAO. The amplitude calibration is estimated to be uncertain at the $10 \%$ level and we have scaled the flux densities of components $\mathrm{A}$ and $\mathrm{B}$ by factors of 1.10 and 1.05 to make them better fit the extrapolation of the flux densities measured at larger wavelengths. The phase noise rms was about $15^{\circ}$, indicating good weather conditions. The phase center of these observations was at $\alpha(2000)=18^{\mathrm{h}} 53^{\mathrm{m}} 18.7 ; \delta(2000)=+01^{\circ} 14^{\prime} 58^{\prime \prime}$.

These data were acquired and reduced using the recommended VLA procedures for high-frequency data, including the fast-switching mode with a cycle of $120 \mathrm{~s}$. The effective bandwidth of the observations was $100 \mathrm{MHz}$. We made images from the calibrated visibilities using weights that were intermediate between natural and uniform (with the ROBUST parameter of the task IMAGR set to 0). The resulting images had an angular resolution of 0 .' $049 \times 0$. .'041; P.A. $=-5^{\circ}$, and we convolved them to a circular angular resolution of $0 .{ }^{\prime \prime} 05$. These images have an rms noise of $0.15 \mathrm{mJy}^{\text {beam }^{-1}}$.

\section{MODELS FOR THE IONIZED GAS}

The maps are shown in Figure 1. The total flux densities at $7 \mathrm{~mm}$ of sources A and B are $120 \pm 6$ and $126 \pm 6 \mathrm{mJy}$, respectively. Gaussian ellipsoid fits to the sources gave angular dimensions of $0^{\prime \prime} .54 \pm 0^{\prime \prime} .02 \times 0^{\prime \prime} .40 \pm 0^{\prime \prime} .01$; P.A. $=178^{\circ} \pm 4^{\circ}$ and $0^{\prime \prime} .39 \pm 0^{\prime \prime} .01 \times 0^{\prime \prime} .32 \pm 0^{\prime \prime} .01$; P.A. $=15^{\circ} \pm 5^{\circ}$ for sources $\mathrm{A}$ and $\mathrm{B}$, respectively. Although the structure is clumpy, both maps suggest limb brightening. This figure shows that the intensity from the shells is not uniform, showing variations of an order of $1 \mathrm{mJy}$ beam $^{-1}$, even over angular scales as small as the beam. Since the rms noise is much smaller $\left(0.15 \mathrm{mJy}^{\text {beam }}{ }^{-1}\right)$, we conclude that these variations are real (and not due to noise) and that most probably the shells are clumpy. Nevertheless, for simplicity, we will consider models with smooth density gradients. In this work we consider shell models bounded by
Table 1

Model Parameters

\begin{tabular}{ccccc}
\hline \hline & $\alpha$ & $A\left(\mathrm{AU}^{\alpha} \mathrm{cm}^{-3}\right)$ & $R_{1}(\mathrm{AU})$ & $R_{2}(\mathrm{AU})$ \\
\hline $\mathrm{G} 34.26+0.15 \mathrm{~A}$ & $0.3_{-0.3}^{+0.4}$ & $1.24_{-0.38}^{+0.13} \times 10^{6}$ & 400 & $1150_{-100}^{+200}$ \\
$\mathrm{G} 34.26+0.15 \mathrm{~B}$ & $1.0_{-0.7}^{+0.5}$ & $1.48_{-0.54}^{+0.54} \times 10^{8}$ & 400 & $1000_{-50}^{+100}$ \\
\hline
\end{tabular}

an inner radius, $R_{1}$, and an outer radius, $R_{2}$, with power-law density $n_{e}(r)=A r^{-\alpha}$, where $A$ is the normalization constant. The electron density is taken to be zero outside the shell. Thus, to compare with the observed $7 \mathrm{~mm}$ intensity profiles, the model intensity profiles are convolved with a circular Gaussian beam with the full width at half-maximum (FWHM) equal to $\theta_{\text {beam }}=0^{\prime \prime} .05$. Given the inner radius $R_{1}=400 \mathrm{AU}$ estimated from the position of the peak of the averaged intensity profiles (Figure 2), we find the parameters $R_{2}, A$, and the density slope $\alpha$ that best fit the observations.

Figure 2 shows the averaged intensity profiles of G34.26+0.15 A and B (solid circles), together with the model intensity profiles for the parameters found to give the best fit for each source (solid line). The intensity averages and rms values were obtained over circular, concentric annuli of width 0.02 each, using the task IRING of AIPS. Since our angular resolution is $00^{\prime} 05$, the averages are not fully independent of each other. The intensity at the peak is $2 \sigma$ and $3 \sigma$ higher than the intensity at the center of sources A and B, respectively. The values of the model parameters that best fit the profiles and the source spectra are shown in Table 1 . The best-fit values were obtained from a $\chi^{2}$ test, where the minimum value of $\chi^{2}$ for both the $7 \mathrm{~mm}$ intensity profile and the spectral energy distribution was found for each source. The exploration of the parameter's space on the $\chi^{2}$ test included density gradients with slopes $0 \leqslant \alpha \leqslant 2.5$ and external radii $500 \leqslant R_{2} \leqslant 1500 \mathrm{AU}$. The errors of the parameters quoted in Table 1 correspond to the size of the $1 \sigma$ volume of the $\chi^{2}$ isocontours in the parameter space. The best-fit models have shallow density gradients $(\alpha)$ which fit the FWHM of 

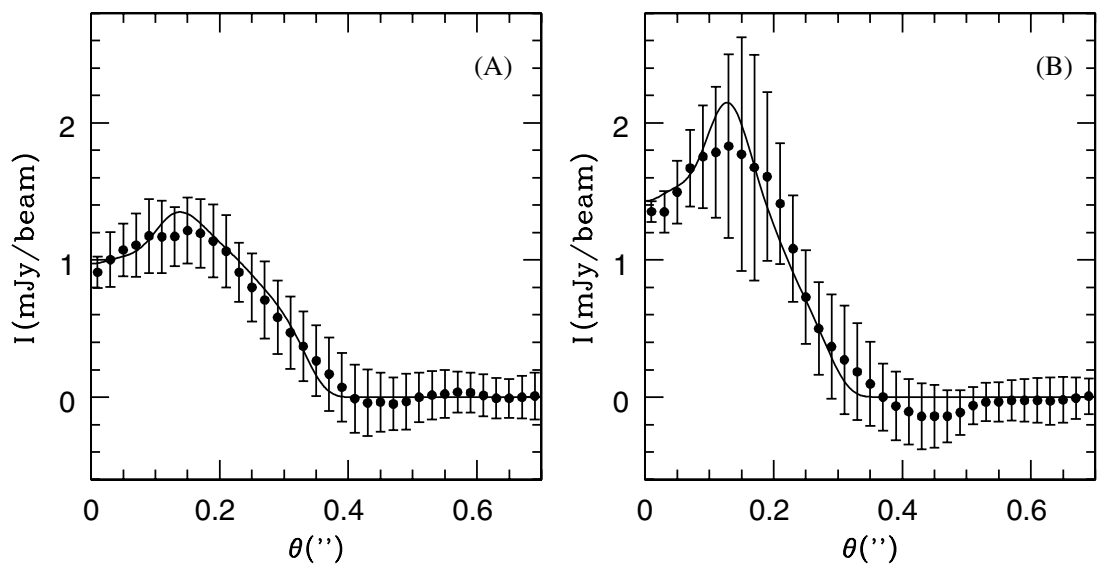

Figure 2. Intensity profiles at $7 \mathrm{~mm}$ of G34.26+0.15 A and B. The solid circles correspond to the observations. The solid lines correspond to the best-fit model for each source (see Table 1).

the profiles. The other free parameters $A$ and $R_{2}$ are necessary to produce the correct intensity normalization and energy flux at low frequencies, respectively. We found that steep density gradients $(\alpha \sim 2)$, allowed in the analysis of A2006 regarding unresolved observations at several frequencies, produce too narrow intensity profiles which do not agree with the observations. This is shown in Figure 3. The continuous line corresponds to the model for source A in Table 1 with $\alpha=0.3$. The broken line corresponds to a model with $\alpha=2, R_{1}=550 \mathrm{AU}$, $R_{2}=1230 \mathrm{AU}, A=1.1 \times 10^{11} \mathrm{AU}^{\alpha} \mathrm{cm}^{-3}$. The main difference is the FWHM angular size of the profiles: steep density profiles produce narrow intensity profiles and shallow density profiles produce wider intensity profiles. Uniform density cores were discarded because they could not fit the observations within the error bars. The main reason for this is that the intensity profile for a model must remain at least almost flat for the first 0 !' 15 to reproduce the observations; instead, for a uniform core model the intensity decreases rapidly from the center to the sides.

Figure 4 shows the spectra of sources G34.26+0.15 A and B. The solid circles correspond to the observations. The solid line in each panel corresponds to the models in Table 1. Figure 5 shows the observed angular sizes (solid circles) and the angular sizes of the shell models (open triangles). To compare the model angular size with the observed size at each frequency, the model intensity, $I_{\nu}$, is convolved with a circular Gaussian beam, $\theta_{\text {beam }}$, with the FWHM equal to the geometric average of the major and minor axes of the beams (see Table 1 of A2006 for the beam sizes). Because the intensity profiles obtained from the $7 \mathrm{~mm}$ observations show that the profiles are not Gaussian, the sizes of the sources at $7 \mathrm{~mm}$ for both the observations and the models are taken as the full width at which the intensity is half the maximum value of the intensity profile. Figure 5 shows that the angular sizes of the shell models are in good agreement with the observed angular sizes.

\section{DISCUSSION}

The high-resolution $7 \mathrm{~mm}$ observations of the $\mathrm{HC} \mathrm{H}$ II regions G34.26+0.15 A and B indicate possible limb brightening, which supports the idea of inner holes in the electron density distribution. It is possible to reproduce these profiles with shell models that have inner radii $R_{1} \sim 400 \mathrm{AU}$. The models that best fit the $7 \mathrm{~mm}$ profiles have density gradients with shallow slopes $(\alpha \sim 0.3-1.0)$. Figure 3 shows that steeper slopes

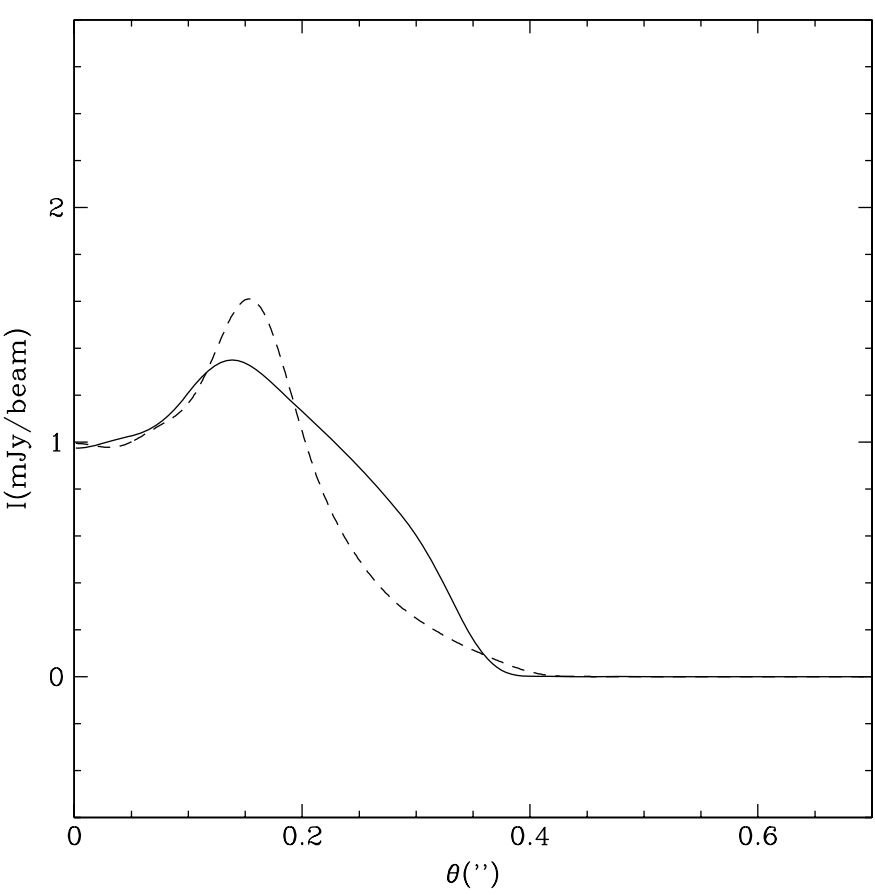

Figure 3. Intensity profiles of models with different density slopes. Solid line: $\alpha=0.3$. Dashed line: $\alpha=2$.

produce profiles which are too narrow to fit the observations. Note that the shallow slopes can trap the ionization front at $R_{2}$, which has values of $\sim 1000 \mathrm{AU}$ for these sources. In contrast, for steep slopes $\alpha>3 / 2$ the ionization front can escape from the cloud (Franco et al. 1990). Our models also reproduce the observed spectra and angular sizes as a function of frequency.

Recently, Beltrán et al. (2007) found a shell-like structure for the HC H II region G24.78+0.08 A1 with high-resolution $7 \mathrm{~mm}$ VLA observations similar to those presented here. This source is found at $7.7 \mathrm{kpc}$, twice the distance to the G34.26+0.15 complex. The intensity profile shown in their Figure 4 is very similar to the profiles in Figure 2. For G24.78+0.08 A1 the angular distance between the maxima is $\sim 0^{\prime \prime} 09$ which corresponds to $\sim 700 \mathrm{AU}$. For G34.26+0.15 A and B, the angular distance between the maxima is $\sim 00^{\prime} 24$ which corresponds to $\sim 800 \mathrm{AU}$. The ratio between the intensity at the maxima and at the center is $\sim 1.3$ for all three sources. The FWHMs of the HC H II regions 

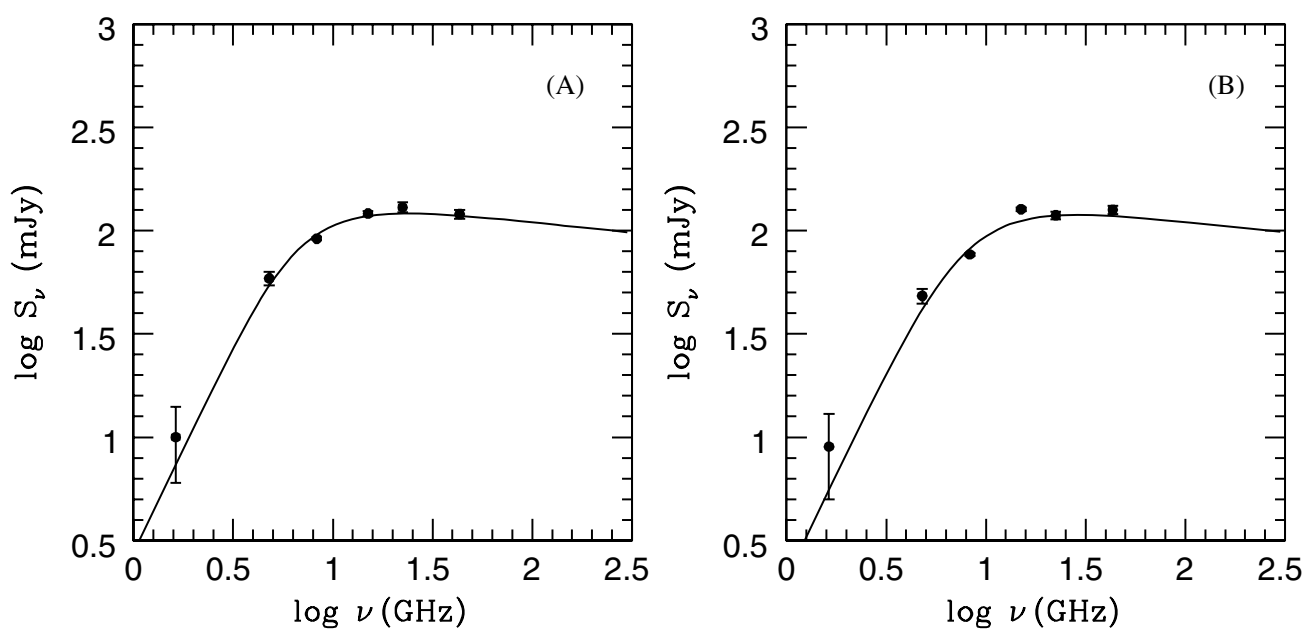

Figure 4. Radio continuum spectra of G34.26+0.15 A and B. The solid circles correspond to the observations. The solid lines correspond to the models discussed in Section 3 (see Table 1).
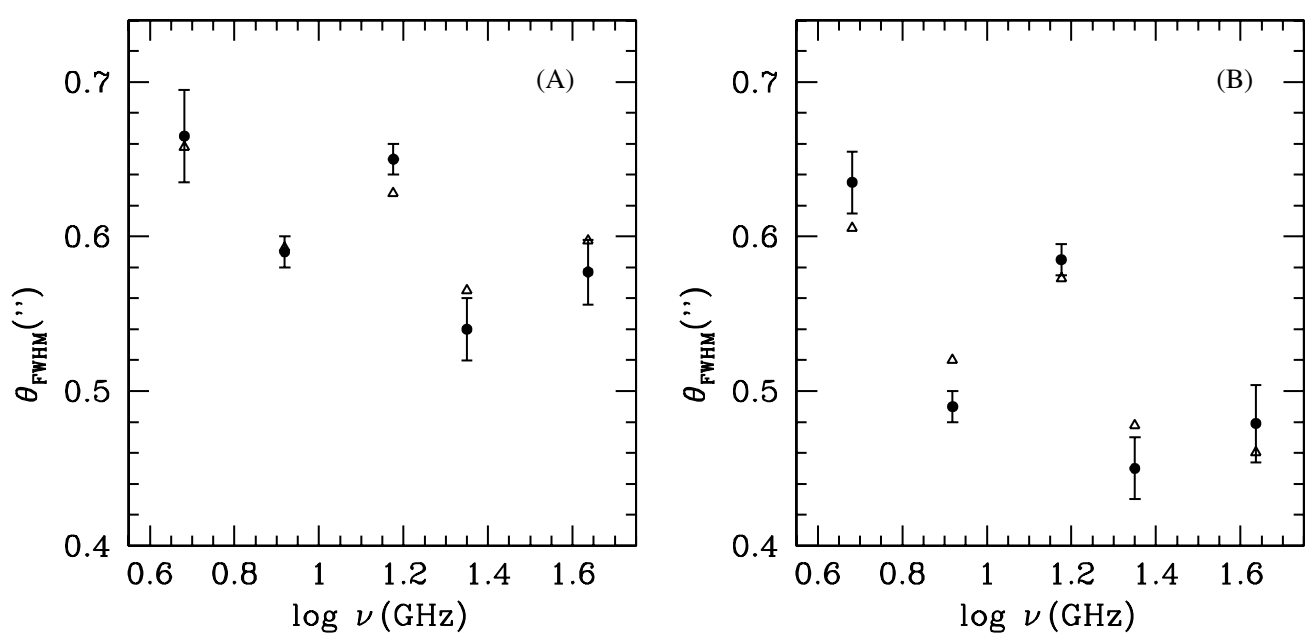

Figure 5. FWHM angular sizes as a function of frequency of G34.26+0.15 A and B. The solid circles correspond to the observations. The open triangles correspond to the shell models.

are $1300 \mathrm{AU}$ for $\mathrm{G} 24.78+0.08 \mathrm{~A} 1,2100 \mathrm{AU}$ and $1700 \mathrm{AU}$ for $\mathrm{G} 34.26+0.15 \mathrm{~A}$ and $\mathrm{B}$, respectively. The calculated rate of ionizing photons for $\mathrm{G} 24.78+0.08 \mathrm{~A} 1$ is $6.7 \times 10^{47} \mathrm{~s}^{-1}$, and it is of the order of $1.4 \times 10^{47} \mathrm{~s}^{-1}$ for both G34.26+0.15 A and B, a factor of the order of 4 lower for the latter sources. These ionizing photon rates correspond to early-B-type stars, with a difference of one spectral type earlier for G24.78+0.08 A1. These estimates neglect the absorption of ionizing photons by dust inside the H II region. The emission measures of the three sources are very similar, EM $\sim 1-4 \times 10^{8} \mathrm{~cm}^{-6} \mathrm{pc}$, on the lower side of emission measures of $\mathrm{HC} \mathrm{H}$ II regions.

Beltrán et al. (2007) interpreted the intensity profile of $\mathrm{G} 24.78+0.08 \mathrm{~A} 1$ as arising from a uniform density thin ionized ring with $R_{2}=590 \mathrm{AU}$ and $R_{1} \sim 0.9 R_{2}$. It is possible to reproduce the observed $7 \mathrm{~mm}$ intensity profile with a thicker ionized shell and a shallow density gradient such as those in G34.26+0.15 A and B. However, we are able to reproduce only the $7 \mathrm{~mm}$ and $1.3 \mathrm{~cm}$ fluxes, obtained at the same time as the $7 \mathrm{~mm}$ intensity profile. This may be due to the variability of the flux of the source, which was recently reported by Galván-Madrid et al. (2008). In this case, the spectral energy distribution obtained from the model should only be compared with fluxes observed at the same time. New high-resolution observations of G24.78+0.08 A1 done simultaneously at both high and low frequencies are needed in order to compare with different models. A very interesting observation of G24.78+0.08 A1 is the presence of proper motions of water masers at the outer edge of the ionized gas (Moscadelli et al. 2007). This beautiful result shows that the HC HII region is surrounded by an expanding molecular shell. It would be interesting to try similar observations for sources G34.26+0.15 A and B.

Sewiło (2006) also found a shell-like structure for the HC H II region G28.20-0.04N with high resolution $7 \mathrm{~mm}$ VLA observations. This source has a rate of ionizing photons greater than $10^{48} \mathrm{~s}^{-1}$, a factor of 10 higher than sources G34.26+0.15 A and $\mathrm{B}$, and the angular distance between the maxima is $\sim 0^{\prime \prime} .36$ which corresponds to $2040 \mathrm{AU}$ at a distance of $5.7 \mathrm{kpc}$. Highresolution radio recombination line observations indicate a rotating torus with an inner radius of $1100 \mathrm{AU}$, an outer radius of $2500 \mathrm{AU}$, and an $\mathrm{EM} \sim 10^{9} \mathrm{~cm}^{-6} \mathrm{pc}$ (Sewiło et al. 2008). Thus, this source is ionized by a higher-mass star and has a larger size than the sources G34.26+0.15 A and B and G24.78+0.08 A1.

An important question, which is still unanswered, is the origin of the inner holes or the thick shells. As mentioned by A2006, slow disk winds may have cleared an inner region (e.g., Lugo et al. 2004). Also, thick ionized shells are expected to form 
in the early stages of massive star formation before the star turns on a strong wind (Yorke \& Kruegel 1977). Once the fast stellar wind starts, it produces very thin shells (see, e.g., Castor et al. 1975; Shull 1980). Instead, the four sources discussed above have fractional widths, $\left(R_{2}-R_{1}\right) / R_{2} \sim 0.1-0.6$, which are much larger than ionized shells swept up by stellar winds. It is interesting to remark that the four shell $\mathrm{H}$ II regions studied by Carral et al. (2002), with radii in the $0.06-1.75 \mathrm{pc}$ range, show similar fractional widths, in the range of $\sim 0.2-0.4$. Another possibility is that the observed ionized gas corresponds to a mass-loaded stellar wind such as those studied by Lizano et al. (1996). In this latter case, the ionization front is trapped in a mass-loaded stellar wind. Scaled to the high densities of $\mathrm{HC} \mathrm{H}$ II regions, these models predict fractional widths $\sim 0.4$, in agreement with our results. This model predicts the existence of blueshifted atomic gas expanding at the speed of sound of the ionized gas, in absorption against the radio continuum emission of the H II region. It is unclear if molecules could form in this warm dense gas to account for the expanding molecular shell observed in G24.78+0.08 A1. More observations are needed to understand the nature of the observed thick shells in the $\mathrm{HC} \mathrm{H}$ II regions.

\section{CONCLUSIONS}

VLA $7 \mathrm{~mm}$ high-resolution observations of G34.26+0.15 $\mathrm{A}$ and $\mathrm{B}$ indicate possible limb-brightened profiles. These profiles would correspond to $\mathrm{HC} \mathrm{H}$ II regions with large inner holes (with inner radii $R_{1} \sim 400 \mathrm{AU}$ ). Thus, to interpret these observations, we consider shell models with power-law electron density gradients. We find that the exponent of the power-law density is a key parameter to reproduce the observed intensity profiles. The observed profiles discriminate against models with steep density gradients that produce profiles too narrow to fit the observations. Instead, shallow gradients with density exponents $\alpha \sim 0.3-1.0$ are preferred. Models for both sources have thick shells that are much larger than those of ionized shells swept up by stellar winds.
It is interesting to note that other $\mathrm{HC} \mathrm{H}$ II regions also seem to have this thick shell morphology: G24.78+0.08 A1 (Beltrán et al. 2007) and G28.20-0.04 N (Sewiło et al. 2008). The physical origin of these thick ionized shells is not yet known but it would be important to find out if this is a pervasive characteristic at this stage of development of $\mathrm{H}$ II regions. An observational program of high-frequency high angular resolution radio observations is required to address this issue.

M.A., S.L., and L.F.R. are grateful to DGAPA/UNAM and CONACyT, México for their support.

\section{REFERENCES}

Avalos, M., Lizano, S., Rodríguez, L. F., Franco-Hernández, R., \& Moran, J. M. 2006, ApJ, 641, 406,(A2006)

Beltrán, M. T., Cesaroni, R., Moscadelli, L., \& Codella, C. 2007, A\&A, 471, L13

Campbell, M. F., Garland, C. A., Deutsch, L. K., Hora, J. L., Fazio, G. G., Dayal, A., \& Hoffmann, W. F. 2000, ApJ, 536, 816

Carral, P., Kurtz, S. E., Rodríguez, L. F., Menten, K., Cantó, J., \& Arceo, R. 2002, AJ, 123, 2574

Castor, J., McCray, R., \& Weaver, R. 1975, ApJ, 200, L107

Franco, J., Tenorio-Tagle, G., \& Bodenheimer, P. 1990, ApJ, 349, 126

Galván-Madrid, R., Rodríguez, L. F., Ho, P. T. P., \& Keto, E. 2008, ApJ, 674, L33

Hoare, M. G., Kurtz, S. E., Lizano, S., Keto, E., \& Hofner, P. 2007, in Protostars and Planets V, ed. B. Reipurth, D. Jewitt, \& K. Keil (Tucson, AZ: Univ. Arizona Press), 181

Lizano, S., Canto, J., Garay, G., \& Hollenbach, D. 1996, ApJ, 468, 739

Lugo, J., Lizano, S., \& Garay, G. 2004, ApJ, 614, 807

Moscadelli, L., Goddi, C., Cesaroni, R., Beltrán, M. T., \& Furuya, R. S. 2007, A\&A, 472, 867

Panagia, N. 1973, AJ, 78, 929

Sewiło, M. M. 2006, PhD thesis, The Univ. of Wisconsin-Madison

Sewiło, M., Churchwell, E., Kurtz, S., Goss, W. M., \& Hofner, P. 2004, ApJ, 605,285

Sewiło, M., Churchwell, E., Kurtz, S., Goss, W. M., \& Hofner, P. 2008, ApJ, 681,350

Shull, J. M. 1980, ApJ, 238, 860

Vacca, W. D., Garmany, C. D., \& Shull, J. M. 1996, ApJ, 460, 914

Yorke, H. W., \& Kruegel, E. 1977, A\&A, 54, 183 